\title{
Equalization in Amplify-Forward Full-Duplex Relay with Direct Link
}

\author{
Karra Chinmay Dheeraj, Andrew Thangaraj, Radhakrishna Ganti \\ Department of Electrical Engineering, \\ Indian Institute of Technology Madras, Chennai, India. \\ Email: $\{$ dheeraj.k, andrew, rganti\}@ee.iitm.ac.in
}

\begin{abstract}
An ideal full-duplex relay doubles the achievable data rate compared to a half-duplex relay. However, in practice, the self-interference and processing delay induces an ISI channel between the source and the destination nodes. In this paper, we study the outage performance of a full-duplex relaying network with amplify-and-forward scheme. In contrast to prior work, we include the direct link from the source to destination, and analyze the distribution of the end-to-end signal-to-noise ratio (SNR) with the minimum mean squared error decision feedback equalizer. We observe that the direct link provides a significant SNR gain, and including it is particularly important for self-interference combating at the receiver.
\end{abstract}

\section{INTRODUCTION}

Full-duplex relaying is a lucrative way of doubling the achievable rate compared to a half-duplex relay. There has been substantial work on ideal full-duplex relays, which can transmit and receive at the same frequency and same time. However, current full-duplex nodes [1], [2] are far from being ideal. While current implementations achieve almost $80-100 \mathrm{~dB}$ cancellation, because of the analog and RF circuit imperfections, the transmit signal cannot be canceled entirely and interferes with the received signal. This interference from the relay node's own transmitting signal is termed as residual self-interference (RSI).

While relaying with an ideal full-duplex node (relay) has been well understood, the analysis of non-ideal full-duplex relaying is complicated because of self-interference. Even with flat-fading channels, the self-interference induces an ISI channel, thus reducing the effective signal-to-noise ratio (SNR) [3] at the destination. Hence with self-interference, it is intuitive that the direct link from source to destination can aid in improving the effective SINR. In this paper, we analyze a relay network with a non-ideal full-duplex relaying node along with a direct source-destination link.

Related work: The probability density function (PDF) of SNR in a half-duplex relay (HDR) network has been obtained in [4] for the amplify and forward scheme, using which system metrics like outage probability and ergodic capacity are calculated.

Mode selection between full-duplex nodes and half-duplex nodes has been considered in [5] with the amplify-and-forward protocol. A trade-off between the self-interference and the spectral efficiency is obtained, using which the maximum tolerable self-interference (compared to the performance of a 978-1-4799-6619-6/15/\$31.00 (c) 2015 IEEE

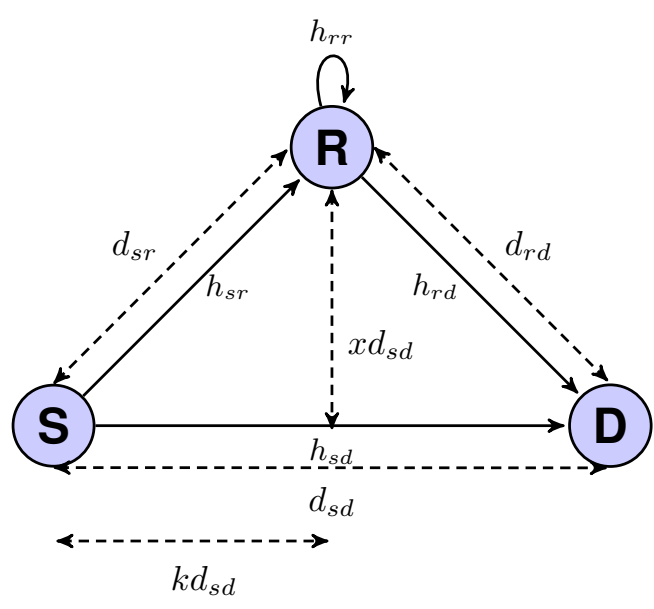

Figure 1: AF Full Duplex Relay Model.

HDR) is computed. Full-duplex relay networks with decodeand-forward protocol is considered in [6]. However instantaneous self-interference cancellation is assumed, which is not true in practice. In [3], processing delay is included which leads to an ISI channel when FDR is used. MMSE-DFE equalization is used to remove the ISI. However, direct link is neglected for the equalization. Two way full-duplex relaying with optimal power allocation is considered in [7], where the source, the relay and the destination are assumed to be fullduplex nodes.

In this paper, we consider a full-duplex relay network with processing delay along with the direct link. Due to the processing delay at the relay node and the self-interference, the equivalent channel between the source and destination is shown to be an ISI channel. We obtain the exact distribution of the effective SNR at the receiver when ISI is considered as noise. We also obtain bounds on the SNR distribution with the MMSE-DFE equalizer.

The rest of the paper is organized as follows: In Section II, the system and signal model are described. In Section III, analysis is carried out treating the RSI as noise. In Section IV, the equalization of RSI with the direct link is considered. 


\section{System AND Signal Model}

\section{A. System Model and Notation}

We consider a source, a full-duplex relay (FDR) and a destination as in Fig. 1. The destination is at a distance $d_{s d}$ from the source. The horizontal distance of the relay from the source is taken to be $k d_{s d}$ where $0<k<1$ and the vertical distance of the relay is taken to be $x d_{s d}$ where $x>0$. The channels from source to destination $h_{s d}$, source to relay $h_{s r}$, relay to destination $h_{r d}$ and the self interference channel from relay to relay $h_{r r}$ are modeled as complex Gaussian channels with zero mean and variance $\sigma_{i j}^{2}$ i.e. $\sim \mathcal{C N}\left(0, \sigma_{i j}^{2}\right)$ where $i \in\{s, r\}, j \in\{r, d\}, i \neq j$. The variance is modeled as $\sigma_{i j}^{2}=c d_{i j}^{-\alpha}$ where $c$ is constant, $\alpha$ is the path loss exponent and $d_{i j}$ is the distance between node $i$ and $j, i \neq j$. The variance of the relay self-interference channel $h_{r r}$ is denoted $\Omega_{r}$.

The symbols are sent in a block, and the channels described above remain constant for the entire block. The relay is assumed to know the gain $h_{s r}$ and $h_{r r}$. Similarly, the destination is assumed to know the channel gains $h_{s d}, h_{r r}$ and $h_{r d}$. The noise added at the relay $n_{r}[n]$ and at the destination $n_{d}[n]$ are complex AWGN with zero mean and variance $\sigma^{2}$ i.e. $\sim \mathcal{C N}\left(0, \sigma^{2}\right)$. The average transmit power at the source and the relay are $P_{s}$ and $P_{r}$, respectively.

The signal-to-noise ratio (SNR) of the link from node $i$ to $j$, denoted $\gamma_{i j}$, is seen to be $\gamma_{i j}=P_{i}\left|h_{i j}\right|^{2} / \sigma^{2}$. Clearly, $\gamma_{i j}$ are exponentially distributed with mean $P_{i} \sigma_{i j}^{2} / \sigma^{2}$ for $i \neq j$ and $P_{r} \Omega_{r r} / \sigma^{2}$ for $i=j=r$. The notation $E[$.] denotes the expectation.

\section{B. Signal Model}

The received signal at the relay is

$$
y_{r}[n]=h_{s r} \sqrt{P_{s}} x_{s}[n]+h_{r r} \sqrt{P_{r}} x_{r}[n]+n_{r}[n],
$$

where $x_{s}[n]$ and $x_{r}[n]$ are the transmitted symbols from the source and the relay, respectively, at time $n$. To satisfy the power constraint, we require $E\left[\left|x_{r}[n]\right|^{2}\right]=1$ and $E\left[\left|x_{s}[n]\right|^{2}\right]=1$.

We consider the amplify-forward protocol at the relay with the assumption that the processing delay at the relay is one symbol period. So, the transmitted signal from the relay is

$$
x_{r}[n]=g_{f} y_{r}[n-1],
$$

where the relay gain $g_{f}$ is chosen such that the average transmitter power at the relay is $P_{r}$. From (1), we see that

$$
E\left[\left|y_{r}[n]\right|^{2}\right]=P_{s}\left|h_{s r}\right|^{2}+P_{r}\left|h_{r r}\right|^{2}+\sigma^{2},
$$

where the fact that $E\left[\left|x_{s}[n]\right|^{2}\right]=E\left[\left|x_{r}[n]\right|^{2}\right]=1$ is used. Using (3) in (2), the relay gain is computed to be

$$
g_{f}=\left(\frac{1}{P_{s}\left|h_{s r}\right|^{2}+P_{r}\left|h_{r r}\right|^{2}+\sigma^{2}}\right)^{-\frac{1}{2}} .
$$

Substituting (2) in (1) and the previously received symbols $y_{r}[n-\tau], \tau \geq 1$ recursively, we get that

$$
\begin{aligned}
y_{r}[n]= & \sum_{k=0}^{\infty}\left(\sqrt{P_{s}} h_{s r}\right)\left(\sqrt{P_{r}} g_{f} h_{r r}\right)^{k} x_{s}[n-k] \\
& +\sum_{k=1}^{\infty}\left(\sqrt{P_{r}} g_{f} h_{r r}\right)^{k-1} n_{r}[n-k]+n_{r}[n] .
\end{aligned}
$$

The received signal at the destination $y_{d}[n]=h_{s d} \sqrt{P_{s}} x_{s}[n]+$ $h_{r d} \sqrt{P_{r}} x_{r}[n]+n_{d}[n]$ can be written as

$$
\begin{aligned}
y_{d}[n] & =h_{s d} \sqrt{P_{s}} x_{s}[n] \\
& +\sum_{k=1}^{\infty}\left(\sqrt{P_{r}} g_{f} h_{r d}\right)\left(\sqrt{P_{s}} h_{s r}\right)\left(\sqrt{P_{r}} g_{f} h_{r r}\right)^{k-1} x_{s}[n-k] \\
& +\sum_{k=1}^{\infty}\left(\sqrt{P_{r}} g_{f} h_{r d}\right)\left(\sqrt{P_{r}} h_{s r}\right)\left(\sqrt{P_{r}} g_{f} h_{r r}\right)^{k-1} n_{r}[n-k] \\
& +n_{d}[n],
\end{aligned}
$$

where we have used the expression for $y_{r}[n]$ in (5). The different terms in the expression for $y_{d}[n]$ are readily interpreted as follows. The term $h_{s d} \sqrt{P_{s}} x_{s}[n]$ in the first line is due to the direct link. The term in the second line is the relay selfinterference (RSI) term, which is a filtered version of the signal $x_{s}[n]$ because of the amplify-forward protocol. The third line is the filtered version of the noise at the relay.

\section{TREATING RSI AS NOISE}

In this section, we treat the RSI term in $y_{d}[n]$ as noise in the processing at the receiver. Under this assumption, the average throughput performance of a half-duplex relay (HDR) and a full-duplex relay (FDR) are compared so as to deduce how well FDR performs even by treating RSI as noise.

Using (1) and (2), $y_{d}[n]$ can be written as

$$
\begin{aligned}
y_{d}[n]=h_{s d} & \sqrt{P_{s}} x_{s}[n]+g_{f} h_{r d} \sqrt{P_{r}}\left(h_{s r} \sqrt{P_{s}} x_{s}[n-1]\right. \\
& \left.+h_{r r} \sqrt{P_{r}} x_{r}[n-1]+n_{r}[n-1]\right)+n_{d}[n] .
\end{aligned}
$$

In (7), treating the RSI term $h_{r r} \sqrt{P_{r}} x_{r}[n-1]$ as noise, the end-to-end SNR at the destination, denoted $\gamma_{f}$, is given by

$$
\gamma_{f}=\frac{\left|h_{s d}\right|^{2} P_{s}+g_{f}^{2}\left|h_{s r}\right|^{2}\left|h_{r d}\right|^{2} P_{r} P_{s}}{g_{f}^{2}\left|h_{r d}\right|^{2} P_{r}\left(\left|h_{r r}\right|^{2} P_{r}+\sigma^{2}\right)+\sigma^{2}} .
$$

Substituting the value of relay gain from (4) and expressing in terms of $\gamma_{i j}, i \in\{s, r\}$ and $j \in\{r, d\}$, we get

$$
\gamma_{f}=\frac{\gamma_{s d}\left(\gamma_{s r}+\gamma_{r r}+1\right)+\gamma_{s r} \gamma_{r d}}{\gamma_{r d}\left(\gamma_{r r}+1\right)+\left(\gamma_{s r}+\gamma_{r r}+1\right)} .
$$

We now compute the CDF of $\gamma_{f}$ that will be subsequently used to compute the outage probability and the average throughput.

Lemma 1. The CDF of end-to-end $S N R \gamma_{f}$ is given by $F_{\gamma_{f}}\left(\Gamma_{T}\right)=$

$1-\int_{-1}^{0} \exp \left(\frac{\lambda_{r d} \Gamma_{T}}{\beta}\right) g_{a}(\beta) d \beta-\int_{-1}^{\Gamma_{T}} \frac{\lambda_{r d} g_{a}(\beta) e^{\left(-\lambda_{s d} \Gamma_{T}\right)}}{\beta \lambda_{s d}+\lambda_{r d}} d \beta$

$+\lambda_{r d} \int_{-1}^{0} \frac{e^{\left(\frac{\Gamma_{T}}{a}\left(\beta \lambda_{s d}+\lambda_{r d}\right)\right)-\lambda_{s d} \Gamma_{T}}}{\beta \lambda_{s d}+\lambda_{r d}} g_{a}(\beta) d \beta$, 
where

$$
\begin{gathered}
g_{a}(\beta)=\exp \left(-\frac{\lambda_{s r}\left(\Gamma_{T}-\beta\right)}{\beta+1}\right) \frac{\lambda_{s r} \lambda_{r r}\left(\Gamma_{T}+1\right)}{\lambda_{r r}(\beta+1)+\lambda_{s r}\left(\Gamma_{T}-\beta\right)} \\
{\left[\frac{1}{\lambda_{r r}(\beta+1)+\lambda_{s r}\left(\Gamma_{T}-\beta\right)}+\frac{1}{\beta+1}\right],-1 \leq \beta \leq \Gamma_{T}}
\end{gathered}
$$

and $\lambda_{i j}=\frac{1}{\bar{\gamma}_{i j}}$ for $i \in\{s, r\}, j \in\{r, d\}$.

Proof: See Appendix.

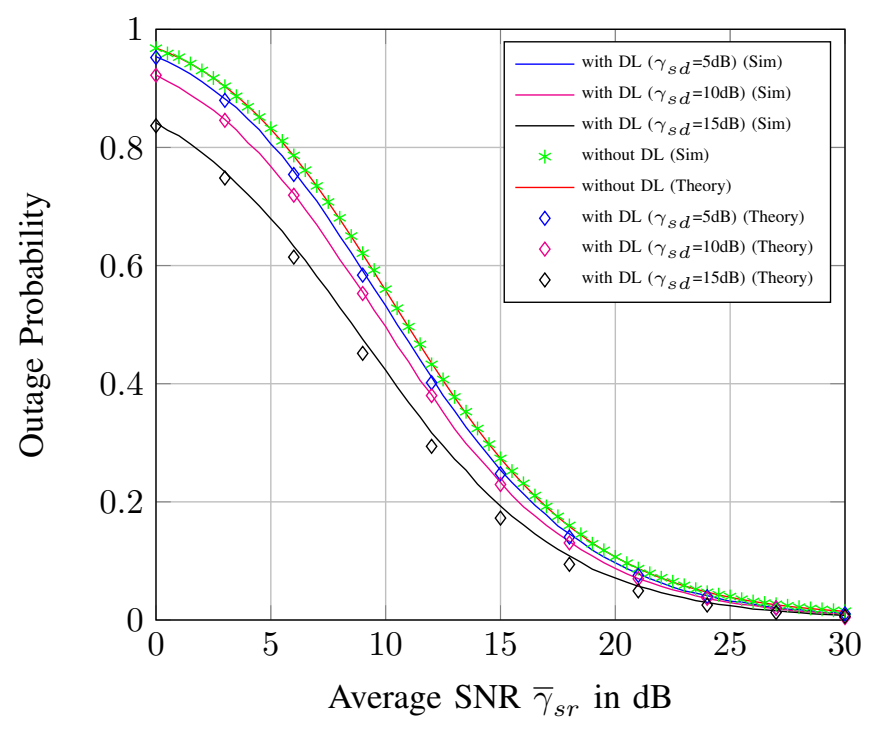

Figure 2: Comparison for different values of $\bar{\gamma}_{s d}$ with $\bar{\gamma}_{r r}=$ $10 \mathrm{~dB}, \bar{\gamma}_{r d}=25 \mathrm{~dB}$ and Rate $=1 \mathrm{bps} / \mathrm{Hz}$.

The outage probability $P\left(\gamma \leq \gamma_{t h}\right)=F_{\gamma}\left(\gamma_{t h}\right)$ is plotted as a function of $\bar{\gamma}_{s r}$ for different values of $\bar{\gamma}_{s d}$. We first observe that the results in Lemma 1 match the Monte-Carlo simulation results. We also observe that the outage probability decreases with increasing strength of the source-destination link.

Using the CDF of $\gamma_{f}$ obtained from Lemma 1 , the average throughput (in nats) is

$$
E\left[\ln \left(1+\gamma_{f}\right)\right]=\int_{0}^{\infty} \frac{1-F_{\gamma_{f}}(t)}{1+t} d t
$$

and can be computed using standard methods of numerical integration.

For the case of half duplex relay (HDR), the average throughput is computed, once again by numerical integration, as $\int_{0}^{\infty} \frac{1}{2} \log _{2}(1+\gamma) f_{\gamma_{h}}(\gamma) d \gamma$, where the PDF of the end-toend SNR $\gamma_{h}$ is [4, eqn. 6]

$$
f_{\gamma_{h}}(\gamma)=\frac{(2 \gamma+1) K_{0}(2 \Gamma)+\left(\lambda_{1}+\lambda_{3}\right) \Gamma K_{1}(\Gamma)}{\lambda_{1} \lambda_{3}} 2 e^{-\frac{\gamma}{\lambda_{1}}-\frac{\gamma}{\lambda_{3}}}
$$

where $K_{v}($.$) is the v$-th order modified Bessel function of the second kind, $\lambda_{1}$ and $\lambda_{3}$ are the means of channels S-R and R-D respectively, and $\Gamma=\sqrt{\left(\gamma^{2}+\gamma\right) / \lambda_{1} \lambda_{3}}$.

In Fig. 3, we show results from simulation as well as numerical calculation of average throughput versus average SNR $\bar{\gamma}_{s r}$ for both FDR and HDR.

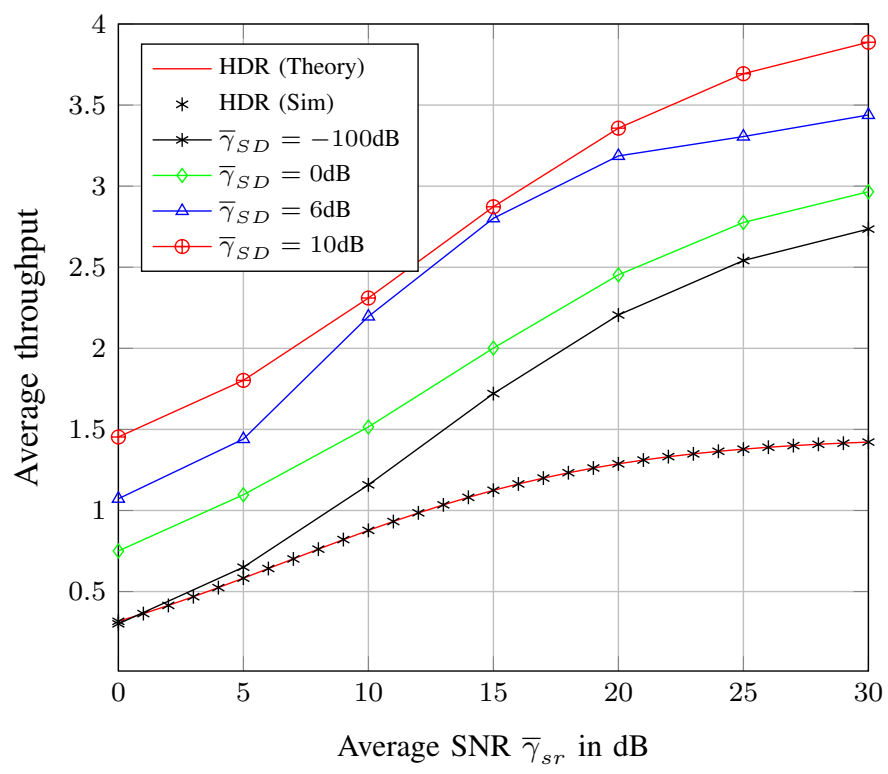

Figure 3: Comparison for different $\bar{\gamma}_{s d}$ with $\bar{\gamma}_{r d}=10 \mathrm{~dB}$ and $\bar{\gamma}_{r r}=5 \mathrm{~dB}$.

From Fig. 3, we see that the average throughput of FDR increases as the average SNR of $\gamma_{s r}$ increases. Also, for the case of FDR, the average throughput increases as the average SNR of $\gamma_{s d}$ increases which indicates that the inclusion of direct link improves the average throughput. This result indicates that the direct link is a significant link in improving the performance of average throughput.

\section{EQUALIZING THE RSI}

In this section, we consider equalization of the RSI term at the destination as opposed to treating it as noise. The destination has the option of either considering or neglecting the direct link term in the equalization. We begin with the case when the direct link is ignored, since this has been considered earlier in the literature [3].

\section{A. No direct link}

The direct link is neglected by setting $h_{s d}=0$ in the signal model. Such a signal model was considered in [3], where the final end-to-end SNR was bounded using an unbiased minimum mean squared error decision feedback equalizer (MMSE-DFE) at the final destination. Denoting the above SNR as $\gamma_{E Q}$, the following bound was established in [3] for a FDR:

$$
\gamma_{E Q} \geq \frac{\gamma_{s r} \gamma_{r d}}{\gamma_{s r}+\gamma_{r d}+2 \gamma_{r r}+1}
$$

For a HDR, there is no RSI and the SNR at the destination $\gamma_{h}$ is obtained by substituting $\gamma_{r r}=\gamma_{s d}=0$ in (9). So, we get

$$
\gamma_{h}=\frac{\gamma_{s r} \gamma_{r d}}{\gamma_{s r}+\gamma_{r d}+1}
$$

The average throughput for the FDR and HDR are comparatively shown in Fig. 4 for a specific set of parameters as 
functions of the distance of the relay from the source with uniform power allocation $P_{s}=P_{r}=P$. Also shown for

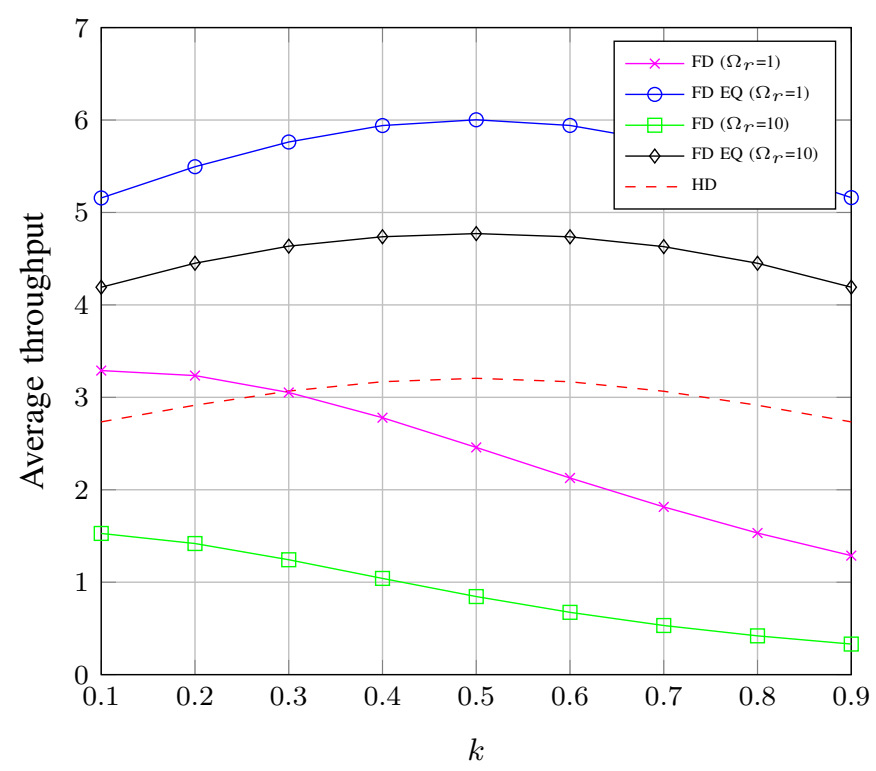

Figure 4: Average throughput comparison for $\Omega_{r}=1,10, d=$ $1, P=20 d B, x=\frac{1}{2}, \alpha=4, c=1, \sigma^{2}=1$.

comparison is the average throughput for the FDR with RSI treated as noise following the method in the previous section. The average throughput is obtained by averaging $\log (1+\gamma)$ over numerous channel realizations.

From Fig. 4, we make the following observations. For the FDR with RSI treated as noise, as the relay moves away from the source, the average throughput decreases, which shows that the source-relay channel is critical and the performance of it depends predominantly on the S-R link. Contrary to this, when the RSI is equalized, the average throughput is symmetrical with respect to mid point of source and destination, which shows that the significant effect of RSI is reduced. Apart from this, the average throughput considering the equalized SNR shows an improvement when compared to those of the unequalized cases. Also as $\Omega_{r}$ increases, the average throughput decreases for both the equalized and unequalized cases.

\section{B. With direct link}

We now consider the case where the direct link is included and the RSI is equalized at the destination. It was shown in [3] that equalizing the RSI at the destination yields better outage probability performance than treating the RSI as noise. But the authors have considered the analysis of outage probability in two cases i.e. without the RSI $\left(h_{r r}=0\right)$ or without the direct link $\left(h_{s d}=0\right)$. The direct link which is another reliable link in sending the symbols from source to destination in the presence of RSI is neglected. In this paper, we consider the case of $h_{r r} \neq 0$ and $h_{s d} \neq 0$, and show that including the direct link for the full duplex relay improves the outage probability performance in the presence of RSI.
From the signal model, the signal and noise power spectral densities, denoted $S_{s}(f)$ and $S_{n}(f)$, respectively, can be obtained as follows:

$$
\begin{aligned}
S_{s}(f)= & P_{s}\left|h_{s d}\right|^{2}+P_{s}\left|h_{s r}\right|^{2}\left|H_{n}(f)\right|^{2} \\
& +2 P_{s} \Re\left\{h_{s d} h_{s r}^{*} H_{n}^{*}(f)\right\}, \\
S_{n}(f)= & \sigma^{2}\left(1+\left|H_{n}(f)\right|^{2}\right),
\end{aligned}
$$

where $\Re\{$.$\} denotes the real part, and$

$$
H_{n}(f)=\sum_{k=1}^{\infty} g_{f} \sqrt{P_{r}} h_{r d}\left(g_{f} \sqrt{P_{r}} h_{r r}\right)^{k-1} \exp (-i 2 \pi f k) .
$$

Using an unbiased minimum mean squared error decision feedback equalizer (MMSE-DFE) [8], the SNR after equalization, denoted $\gamma_{e q}$ is given by

$$
\gamma_{e q}=\exp \left(\int_{-0.5}^{0.5} \log \left[1+\frac{S_{s}(f)}{S_{n}(f)}\right] d f\right)-1 .
$$

Expressing $h_{p q}=h_{p q_{r}}+i h_{p q_{j}}$, where $h_{p q_{r}}$ and $h_{p q_{j}}$ are the real and imaginary parts of the channel $h_{p q}$ for $p \in\{s, r\}$ and $q \in\{r, d\}$ and using the integral in [9, eqn. 4.224.9], we get that

$$
\gamma_{e q}=\frac{Q_{1}+\sqrt{Q_{1}^{2}-Q_{2}^{2}}}{Q_{3}+\sqrt{Q_{3}^{2}-Q_{4}^{2}}}-1,
$$

where

$$
\begin{aligned}
Q_{3}= & \sigma^{2}\left(g_{f}^{2} P_{r}\left|h_{r d}\right|^{2}+1+P_{r}|\theta|^{2}\right), Q_{4}=2 \sqrt{P_{r}}|\theta| \sigma^{2}, \\
Q_{1}= & Q_{3}+P_{s}\left|h_{s d}\right|^{2}\left(1+P_{r}|\theta|^{2}\right)+g_{f}^{2} P_{s} P_{r}\left|h_{s r}\right|^{2}\left|h_{r d}\right|^{2} \\
& -2 P_{s} P_{r} g_{f}^{2}\left(a h_{r r_{r}}-b h_{r r_{j}}\right), \\
Q_{2}= & Q_{4}-2 P_{s} \sqrt{P_{r}}\left(a g_{f}+\left|h_{s d}\right|^{2}|\theta|\right), \theta=g_{f} h_{r r}, \\
a= & h_{s d_{r}}\left(h_{s r_{r}} h_{r d_{r}}+h_{s r_{j}} h_{r d_{j}}\right)+h_{s d_{j}}\left(h_{s r_{r}} h_{r d_{j}}+h_{s r_{j}} h_{r d_{r}}\right), \\
b= & h_{s d_{j}}\left(h_{s r_{r}} h_{r d_{r}}+h_{s r_{j}} h_{r d_{j}}\right)-h_{s d_{r}}\left(h_{s r_{r}} h_{r d_{j}}+h_{s r_{j}} h_{r d_{r}}\right) .
\end{aligned}
$$

Since $\sqrt{Q_{1}^{2}-Q_{2}^{2}} \leq Q_{1}, \gamma_{e q}$ can be upper bounded as,

$$
\gamma_{e q} \leq \frac{2 Q_{1}}{Q_{3}+\sqrt{Q_{3}^{2}-Q_{4}^{2}}}-1 \text {. }
$$

In the expression for $Q_{1}$, the term $a h_{r r_{r}}-b h_{r r_{j}}$ has expected value equal to 0 , and a good, tractable approximation is to set that term equal to zero. Using this approximation, we get

$\gamma_{e q} \approx \frac{2\left(Q_{3}+P_{s}\left|h_{s d}\right|^{2}\left(1+P_{r}|\theta|^{2}\right)+g_{f}^{2} P_{s} P_{r}\left|h_{s r}\right|^{2}\left|h_{r d}\right|^{2}\right)}{Q_{3}+\sqrt{Q_{3}^{2}+Q_{4}^{2}}}-1$.

Substituting the expressions for $Q_{3}, Q_{4}$ and using $\rho=\gamma_{r d}+$ $\gamma_{s r}+2 \gamma_{r r}+1$, we get

$$
\begin{aligned}
\gamma_{e q} & \approx \frac{2\left(\rho+\gamma_{s d}\left(\rho-\gamma_{r d}\right)+\gamma_{s r} \gamma_{r d}\right)}{\rho+\sqrt{\left(\rho-2 \gamma_{r r}\right)^{2}+4 \gamma_{r r} \gamma_{r d}}}-1 \\
& \leq \frac{\gamma_{r r}+\gamma_{s r} \gamma_{r d}+\gamma_{s d}\left(\gamma_{s r}+2 \gamma_{r r}+1\right)}{\gamma_{r d}+\gamma_{s r}+\gamma_{r r}+1}
\end{aligned}
$$

where in the final bound we ignore the term $4 \gamma_{r r} \gamma_{r d}$ in the denominator. 
Using the above approximation for the equalized SNR, the outage probability is approximately calculated as

$$
\begin{aligned}
& P\left(\gamma_{e q} \leq \gamma_{t h}\right) \approx \\
& \quad P\left(\frac{\gamma_{r r}+\gamma_{s r} \gamma_{r d}+\gamma_{s d}\left(\gamma_{s r}+2 \gamma_{r r}+1\right)}{\gamma_{r d}+\gamma_{s r}+\gamma_{r r}+1} \leq \gamma_{t h}\right) .
\end{aligned}
$$

A technique for evaluating (18) in an integral form is provided in Appendix B. The approximate outage probability is plotted versus the average SNR $\bar{\gamma}_{s r}$ in Fig. 5. The threshold $\gamma_{t h}$

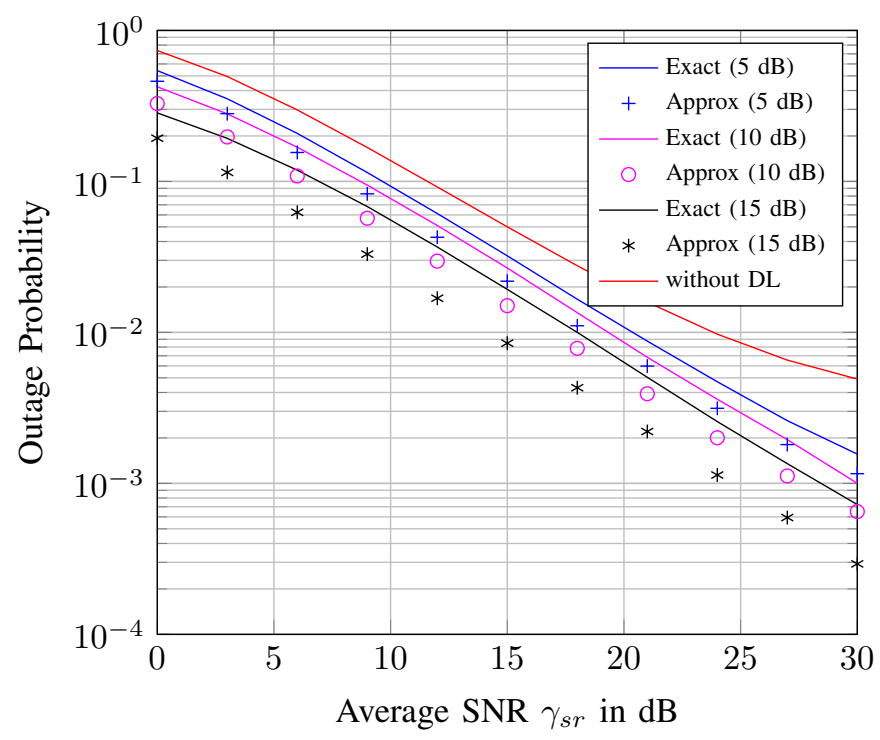

Figure 5: Outage probability for different $\gamma_{s d}$ shown in brackets with $\bar{\gamma}_{r d}=25 \mathrm{~dB}, \bar{\gamma}_{r r}=10 \mathrm{~dB}$ and $R_{o p}=1 \mathrm{bps} / \mathrm{Hz}$.

used for the calculation of outage probability is obtained using $R_{o p}=\log _{2}\left(1+\gamma_{t h}\right)$ where $R_{o p}$ is the rate of transmission for FDR. From Fig. 5, we observe that the approximation for outage probability in (18) is a lower bound to the exact outage probability obtained by simulating the exact $\gamma_{e q}$ in (15) with the bound being fairly close for lower $\gamma_{s d}$. Further, we observe that including the direct link significantly improves the outage performance by $3 \mathrm{~dB}$ at an outage of $10^{-2}$.

The average throughput is shown in Fig. 6 for different transmit power $P$ with uniform power allocation $P_{s}=P_{r}=$ $P$. The average throughput for the cases of equalized SNR with and without direct link are obtained using (17) and (11), respectively. The average throughput for the cases of treating RSI as noise with direct link is obtained using (9). The average throughput for HDR is obtained using (12).

The average throughput of both equalized and unequalized cases for the relay with direct link shows an improvement when compared to those without direct link. The average throughput for the case of equalized RSI with and without direct link outperform the average throughput of HDR for the entire range of transmit power $P$, which is not the case for treating RSI as noise. This shows the importance of equalizing the RSI at the destination in practical FDR systems. Finally, the curves for FDR with direct link and RSI equalized are

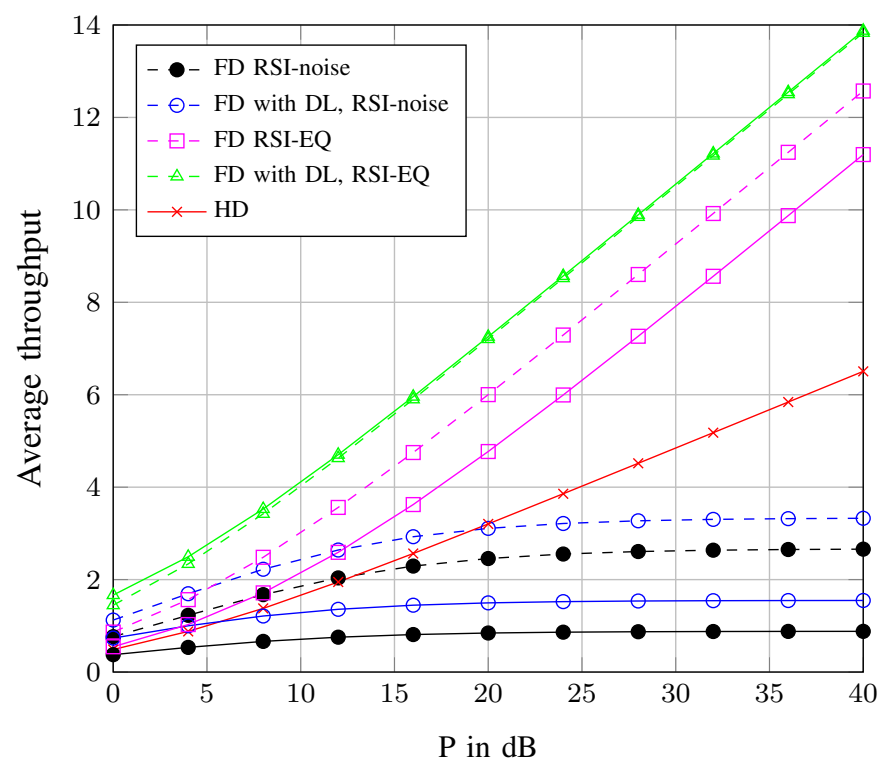

Figure 6: Average throughput comparison: dashed: $\Omega_{r}=1$, solid: $\Omega_{r}=10$. Parameters: $d_{s d}=1, x=\frac{1}{2}, k=\frac{1}{2}, \alpha=$ $4, c=1, \sigma^{2}=1$.

almost overlapping for both $\Omega_{r}=1 \mathrm{~dB}$ and $\Omega_{r}=10 \mathrm{~dB}$, which effectively neutralizes the RSI.

\section{CONCLUSION}

In this paper, we studied a practical full-duplex AF relaying scheme by including both the self-interference and the direct link. The gains from equalizing the resulting ISI at the destination node were characterized by analyzing the distribution of the end-to-end SNR. Substantial gains were seen over comparable half-duplex relaying schemes. This shows that residual self-interference can effectively be compensated by suitable receiver processing at the destination helping to make deployment of full-duplex relays practical.

\section{REFERENCES}

[1] M. Duarte, C. Dick, and A. Sabharwal, "Experiment-driven characterization of full-duplex wireless systems," Wireless Communications, IEEE Transactions on, vol. 11, no. 12, pp. 4296-4307, December 2012.

[2] D. Bharadia, E. McMilin, and S. Katti, "Full duplex radios," in Proceedings of the ACM SIGCOMM 2013 conference on SIGCOMM. ACM, 2013, pp. 375-386.

[3] T. M. Kim and A. Paulraj, "Outage probability of amplify-and-forward cooperation with full duplex relay," in Wireless Communications and Networking Conference (WCNC), 2012 IEEE, 2012, pp. 75-79.

[4] S. Soliman and N. Beaulieu, "Exact analytical solution for dual-hop and opportunistic dual-hop af relaying systems," in Vehicular Technology Conference (VTC Fall), 2012 IEEE, Sept 2012, pp. 1-5.

[5] T. Riihonen, S. Werner, and R. Wichman, "Comparison of full-duplex and half-duplex modes with a fixed amplify-and-forward relay," in Wireless Communications and Networking Conference, 2009. WCNC 2009. IEEE, April 2009, pp. 1-5.

[6] B. Yu, L. Yang, X. Cheng, and R. Cao, "Relay location optimization for full-duplex decode-and-forward relaying," in Military Communications Conference, MILCOM 2013 - 2013 IEEE, Nov 2013, pp. 13-18.

[7] X. Cheng, B. Yu, X. Cheng, and L. Yang, "Two-way full-duplex amplify-and-forward relaying," in Military Communications Conference, MILCOM 2013 - 2013 IEEE, Nov 2013, pp. 1-6. 
[8] J. Cioffi, G. Dudevoir, M. Vedat Eyuboglu, and J. Forney, G.D., "Mmse decision-feedback equalizers and coding. i. equalization results,' Communications, IEEE Transactions on, vol. 43, no. 10, pp. 2582-2594, Oct 1995

[9] I. S. Gradshteyn and I. M. Ryzhik, Table of integrals, series, and products, 7th ed. Elsevier/Academic Press, Amsterdam, 2007.

[10] G. B. Folland, Real analysis: modern techniques and their applications. John Wiley \& Sons, 2013.

\section{APPENDIX A}

\section{PROOF OF LEMMA 1}

Proof: The CDF is given by

$$
F_{\gamma_{f}}\left(\Gamma_{T}\right)=P\left(\gamma_{f} \leq \Gamma_{T}\right)=1-P\left(\gamma_{f}>\Gamma_{T}\right) .
$$

Substituting from (9) for $\gamma_{f}$,

$$
\begin{gathered}
P\left(\gamma_{f}>\Gamma_{T}\right)=P\left(\frac{\gamma_{s d}\left(\gamma_{s r}+\gamma_{r r}+1\right)+\gamma_{s r} \gamma_{r d}}{\gamma_{r d}\left(\gamma_{r r}+1\right)+\gamma_{s r}+\gamma_{r r}+1}>\Gamma_{T}\right) \\
=P\left(\gamma_{s d}>\gamma_{r d}\left(\frac{\Gamma_{T}-\gamma_{R}}{\gamma_{R}+1}\right)+\Gamma_{T}\right)
\end{gathered}
$$

$=E\left[\exp \left(-\lambda_{s d}\left(\gamma_{r d}\left(\frac{\Gamma_{T}-\gamma_{R}}{\gamma_{R}+1}\right)+\Gamma_{T}\right)\right) I_{1}\right]+E\left[1-I_{1}\right]$

where $I_{1}=I\left(\gamma_{r d}\left(\frac{\Gamma_{T}-\gamma_{R}}{\gamma_{R}+1}\right)+\Gamma_{T}>0\right)$ and $I($.$) denotes an$ indicator random variable. The first term in the above equation simplifies to

$$
\lambda_{r d} e^{\left(-\lambda_{s d} \Gamma_{T}\right)} \underset{\gamma_{r d} \beta+\Gamma_{T}>0}{\iint^{-\gamma_{r d}\left(\beta \lambda_{s d}+\lambda_{r d}\right)}} g_{a}(\beta) d \gamma_{r d} d \beta,
$$

where $g_{a}(\beta)$ is the PDF of the random variable

$$
a=\frac{\Gamma_{T}-\gamma_{R}}{\gamma_{R}+1} .
$$

Observe that $-1 \leq a \leq \Gamma_{T}$, and the PDF $g_{a}(\beta)$ given in (10) can be obtained by using the exponential distribution of $\gamma_{s r}$ and $\gamma_{r r}$.

Splitting the integration over the sub-regions $\beta>0$ and $\beta<0$, the integral in (22) equals

$$
\begin{aligned}
& \lambda_{r d} e^{\left(-\lambda_{s d} \Gamma_{T}\right)}\left(\int_{-1}^{\Gamma_{T}} \frac{1}{\beta \lambda_{s d}+\lambda_{r d}} g_{a}(\beta) d \beta\right. \\
& \left.-\int_{-1}^{0} \frac{\exp \left(\frac{\Gamma_{T}}{\beta}\left(\beta \lambda_{s d}+\lambda_{r d}\right)\right)}{\beta \lambda_{s d}+\lambda_{r d}} g_{a}(\beta) d \beta\right) .
\end{aligned}
$$

The second term above can be simplified as

$$
E\left[1-I_{1}\right]=E_{a}\left[\exp \left(\frac{\lambda_{r d} \Gamma_{T}}{a}\right) I(a<0)\right]
$$

which can be written as

$$
E\left[1-I_{1}\right]=\int_{-1}^{0} \exp \left(\frac{\lambda_{r d} \Gamma_{T}}{\beta}\right) g_{a}(\beta) d \beta .
$$

The CDF can be obtained by substituting (24) and (26) in (21).

\section{APPENDIX B}

\section{EVALUATING (18)}

We now express (18) in an integral form. For notational simplicity let $x_{1}=\gamma_{s r}, x_{2}=\gamma_{r d}, x_{3}=\gamma_{s d}$ and $x_{4}=\gamma_{r r}$. Also let $\kappa=\lambda_{1} \lambda_{2} \lambda_{3} \lambda_{4}$ where $\lambda_{i}$ is the inverse of the mean of $x_{i}$. Then the probability in (18) equals

$$
\kappa \int_{X^{T} A X+X^{T} B-T \leq 0} e^{-\Lambda^{T} X} d X
$$

where

$$
A=\left[\begin{array}{cccc}
0 & 2^{-1 / 2} & 2^{-1 / 2} & 0 \\
2^{-1 / 2} & 0 & 0 & 0 \\
2^{-1 / 2} & 0 & 0 & 1 \\
0 & 0 & 1 & 0
\end{array}\right]
$$

and $B=[-T,-T, 1,1-T]^{T}$. Observe that $A$ is a positive definite matrix and, hence, $\sqrt{A}$ exists. Completing the squares, the integral can be rewritten as

$$
P=\kappa \int_{\mathbb{R}^{4}} e^{-\Lambda^{T} X} I_{S}(X) d X,
$$

where $S=\left(\sqrt{A} X+\frac{\tilde{B}}{2}\right)^{T}\left(\sqrt{A} X+\frac{\tilde{B}}{2}\right) \leq \delta^{2}$ and $X=$ $\left[x_{1}, x_{2}, x_{3}, x_{4}\right]^{T}$. Here $\delta^{2}=T+\frac{\|\tilde{B}\|^{2}}{4}$ and $\tilde{B}=\sqrt{A}^{-1} B$. From [10], the above expression equals

$$
P=\kappa \int_{\mathbb{R}^{4}} \operatorname{Real}\left\{\widehat{e^{-\Lambda^{T} X}}(\omega) \widehat{I_{S}(X)}(\omega)\right\} d \omega,
$$

where $\widehat{f(x)}(\omega)$ represents the Fourier transform of the function $f(x)$. We just use the real part, since the final probability is a real number (the imaginary part of the integral can be shown to be 0 ). The Fourier transform of the first term is

$$
\widehat{e^{-\Lambda^{T} X}}(\omega)=\prod_{m=1}^{4} \frac{1}{j \omega_{i}+\lambda_{i}} .
$$

The Fourier transform of the second term is

$$
\widehat{I_{S}(X)}(\omega)=\int_{\left(\sqrt{A} X+\frac{\tilde{B}}{2}\right)^{T}\left(\sqrt{A} X+\frac{\tilde{B}}{2}\right) \leq \delta^{2}} e^{-j<w, x>} d x .
$$

Using the substitution $X=A^{-1 / 2}\left(\delta Y-\frac{\tilde{B}}{2}\right), \widehat{I_{S}(X)}(\omega)$ equals

$$
\delta^{2} \operatorname{det}\left(A^{-1 / 2}\right) e^{j<\omega, A^{-1 / 2} \frac{\tilde{B}}{2}>} \int_{Y^{T} Y \leq 1} e^{-j<A^{-1 / 2} \delta \omega, Y>} d y .
$$

Using the Fourier transform of the indicator of the unit ball in 4 dimensions, we have

$$
\begin{aligned}
& \widehat{I_{S}(X)}(\omega)= \\
& \delta^{2} \operatorname{det}\left(A^{-1 / 2}\right) e^{j<\omega, A^{-1 / 2} \frac{\tilde{B}}{2}>}\left\|A^{-1 / 2} \delta \omega\right\|^{2} J_{2}\left(\left\|A^{-1 / 2} \delta \omega\right\|\right),
\end{aligned}
$$

where $J_{2}(x)$ is the Bessel function. Using the above expression in (27), results in an integral expression for (18). 